REVIEW

OF GLOBAL

MANAGEMENT

Ensayos y Artículos de investigación

FACULTAD DE

NEGOCIOS

UPC

\title{
RETOS PARA LA GESTIÓN Y LA DOCENCIA EN LA EDUCACIÓN SUPERIOR DE AMÉRICA LATINA
}

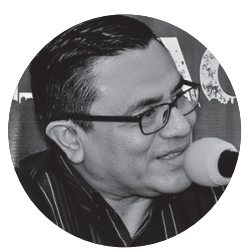

\author{
J. Paul Llaque \\ ORCID: 0000-0001-9037-541X \\ Profesor de Protocolo de Investigación \\ Carrera de Administración \\ Facultad de Negocios, UPC \\ Lima - Perú \\ paul.llaque@gmail.com \\ Recibido: 31 de agosto de 2018 \\ Aprobado: 09 de noviembre de 2018 \\ Cómo citar este artículo: \\ Llaque, P. (2018). Retos para la gestión y la docencia en la educación superior \\ de América Latina. Review of Global Management, 4(2), 54-63.
}

RESUMEN

A inicios de la última década del siglo XX, la gestión y el ejercicio de la docencia universitaria se vieron institucional y continentalmente cuestionados. El denominado proceso de Bolonia impactó profundamente en la educación superior no solo de Europa, el continente en el que se originó, sino de América Latina, mediante dos efectos superlativos: el cambio del rol tradicional del docente y las demandas políticas globales y locales. Frente a la interrogante de qué implicaron dichos efectos, la tesis que guía las líneas siguientes es que esos efectos implicaron e implican cinco principales retos para la gestión y el ejercicio docente en los procesos de educación superior: i) la incorporación del factor productivo basado en el crecimiento y manejo de la información en el desarrollo socioeconómico; ii) las nuevas tecnologías de la información y comunicación como factor acelerador y modificador de los procesos de manejo de información y de las comunicaciones; iii) un nuevo perfil profesional basado en competencias y metas educativas; iv) un nuevo paradigma en el que el estudiante ocupa el centro en el proceso enseñanza - aprendizaje y relega al profesor a un rol de facilitador; $y$ v) el alcance global de la actividad humana que implica la internacionalización de la educación superior. Para probar esta tesis, se adopta una doble perspectiva argumentativa. En primer lugar, el análisis de cada reto; y luego, en segundo lugar, la propuesta o propuestas consecuentes para afrontarlo. Finalmente, se realiza un recuento conclusivo con horizonte de apertura.

Palabras clave: Educación superior, gestión y praxis docente, política educativa.

\section{ABSTRACT}

At the beginning of the last decade of the twentieth century, the business and praxis of university teaching were institutionally and continentally questioned. The so-called Bologna process has had a profound impact on higher education, not only in Europe, where it originated, but 
the change of the traditional role of the teacher, and the global and local political demands. Before the question and communication processes; iii) a new professional obal scope of human activity that implies the internationalization of higher education. To prove this thesis, a double argumentative perspective is adopted. First, an analysis made with an open horizon.

Keywords: Higher education, business and praxis of university teaching, education policy.

\section{INTRODUCCIÓN}

Antes del análisis de cada reto para la gestión y ejercicio de la docencia en la educación superior de América Latina, y de la propuesta consecuente, es necesario cumplir con dos propósitos. El primero es un breve recuento histórico de los proyectos que transformaron la educación superior en Europa y que influyeron en la situación actual de la educación en América Latina. El segundo propósito constituye una explicitación de los marcos de referencia que guían o deben guiar los cambios educativos como consecuencia de los retos.

\subsection{De la Magna Charta Universitatum a Tuning América Latina}

El origen de la transformación contemporánea de la educación superior se gesta en Europa el 18 de setiembre

${ }^{1}$ Programa de Cooperación Académica entre la Unión Europea y América Latina para la Acreditación y Reconocimientos Oficiales entre universidades de Mercosur y la UE.

${ }^{2} 6 \times 4$ se refiere a seis profesiones en cuatro ejes de análisis. UEALC de 1988, con la Declaración de Bolonia. Los rectores de las universidades europeas, firmantes de la denominada Magna Charta Universitatum, "estiman que los pueblos y los Estados han de ser conscientes del papel que las universidades deberían tener en el futuro en una sociedad que se transforma y se internacionaliza" (Declaración de Bolonia, 1988)

Diez años más tarde, en 1998, en la Declaración de La Sorbona, los ministros de Francia, Alemania, Italia y Reino Unido expresaron su deseo de "un sistema de educación superior que ofrezca las mejores oportunidades para buscar y encontrar su propio ámbito de excelencia" (Declaración de la Sorbona, 1998).

Un año más tarde, el 19 de junio de 1999, se estableció la hoy famosa Declaración de Bolonia. Frente a los retos que el mundo globalizado plantea, los ministros europeos de educación se propusieron desarrollar una educación competitiva, moderna y de vanguardia, que respondiera al clima tecnológico y cultural inmediato. En ese evento, se proyectó para 2010 la creación del Espacio Europeo de Educación Superior (EEES) y se agendaron reuniones bienales afines (2001 en Praga, 2003 en Berlín, 2005 en Fregen, 2007 en Londres).

En América Latina, en la Cumbre Birregional de Río de Janeiro, en junio de 1999, los jefes de Estado de la Unión Europea (UE) y de América Latina y el Caribe (ALC) se interesaron en construir un espacio común de enseñanza superior. En 2000, los ministros de educación de la UE y de ALC declararon como urgente la creación de ese espacio común. En 2005, se estableció, en México, que el espacio de educación superior UE-ALC se lograría en diez años a través del proyecto "Horizonte 2015". Asimismo, se concretaron otros proyectos, como el ALFA-ACRO ${ }^{1}$, el UEALC $6 \times 4^{2}{ }^{2}$ y, especialmente, el Alfa Tuning América Latina ${ }^{3}$ (Bugarin Olivera, 2008; Beneitone, Esquetini, González, Maletá, Siufi \& Wagenaar, 2007).

Es importante señalar, sin embargo, que aun cuando "la realidad universitaria actual [de América Latina] dista mucho de ofrecer las condiciones necesarias para la consecución de [los] objetivos [del Proceso de Bolonia]" (Sanjurjo Rivo, 2012, p. 585), resulta impostergable asumir el enfoque y los principios de los grandes gestores del EEES (Declaración de Bolonia, 1988 y 1999; Declaración de

se refiere a la relación entre la Unión Europea y América Latina y el Caribe.

${ }^{3}$ Este proyecto busca "afinar" las estructuras educativas de América Latina. 
La Sorbona, 1998; Comunicado de Londres, 2007), incluso reconociendo que actualmente existe la interrogante sobre "si sería más adecuado en nuestras condiciones contextuales y educativas centrarnos en crear un espacio de educación superior entre ALC, Canadá y Estados Unidos de América" (Bugarin Olivera, 2008, p. 58)

\subsection{Marcos de referencia para el cambio educativo} Las tendencias universales en la educación superior actual constituyen los retos que la gestión y el ejercicio de la docencia deben asumir a fin de lograr una educación superior de mayor calidad. Aun cuando no se ahondó en el análisis de ellas, esas megatendencias o retos, que suscribimos plenamente por considerarlos atinados, fueron establecidos por Tuning América Latina (Beneitone et al., 2007). Producto de la reflexión posterior de un sinnúmero de estudiosos, se han delineado hasta tres marcos de referencia que deben guiar el cambio educativo, a saber: i) políticas educativas internacionales y nacionales; ii) sistemas de aseguramiento de la calidad educativa; $\mathrm{y}$ iii) procesos de armonización de los sistemas de educación superior. En el análisis de cada reto y la consecuente propuesta se tiene en cuenta estos tres marcos de referencia.

\section{LOS CINCO RETOS: ANÁLISIS Y PROPUESTAS CONSECUENTES}

Como ya ha sido dicho en líneas anteriores, las tendencias universales sobre la educación superior en América Latina fueron enunciadas en el informe final del Proyecto Tuning América Latina hace poco más de una década (Beneitone et al., 2007, pp. 23-26). Esas tendencias universales, que también suelen denominarse "megatendencias", constituyen para nosotros los retos que la gestión y la docencia deben afrontar en América Latina para lograr una educación de mayor calidad. A continuación, se enuncia y analiza sucintamente cada reto y luego se explica la propuesta consecuente que, en este artículo, se sugiere para afrontarlo. Asimismo, en algunos acápites se registran algunas implicancias relacionadas específicamente con la educación superior de Chile y del Perú.

\subsection{Primer reto: la incorporación, en el desarrollo económico y social, del factor productivo basado en el conocimiento y manejo adecuado de la información}

De acuerdo con esta tendencia universal, las carreras universitarias deberán concentrarse en la gestión efectiva de la información que les compete o de aquella que las implique, pues la información a secas se ha convertido en un insumo más. Desde antes de la década de 1980, algunos futurólogos, como Alvin Toffler, aseguraban que el conocimiento era poder y que la sociedad del futuro, aquella sociedad en la cual el aprendizaje sería permanente, ya había llegado, y contendía con la sociedad anterior (Toffler, 1979, 1995).

La propuesta consecuente para afrontar este primer desafío es que las universidades adopten como primera o más importante competencia genérica una que incluya la gestión de la información: aprender a aprender. Se debe señalar que, entre las 27 competencias genéricas establecidas por Tuning América Latina, se encuentra la competencia de aprender a aprender con un nombre distinto: "capacidad de aprender y actualizarse"; más aún, dicha competencia figura entre las seis competencias genéricas consideradas más importantes por académicos, graduados, estudiantes y empleadores (Beneitone et al., 2007, pp. 52, 55, 58 y 60; Beneitone, González \& Wagenaar, 2014, p. 82).

\subsection{Segundo reto: las nuevas tecnologías de la información y comunicación (TIC) como factor acelerador y modificador de los procesos de manejo de información y de las comunicaciones}

A nadie escapa que las TICs han cambiado no sólo nuestra manera de informarnos y comunicarnos, sino, incluso, nuestra forma de aprender, de convivir y hasta de pensar. Las nuevas generaciones no han tenido que sufrir la migración al mundo de los bytes y de la Internet, toda vez que son "nativos digitales". Sin embargo, es virtualmente un hecho que hoy cualquier persona letrada y competente se desplaza con relativa comodidad en el universo digital. No obstante ello, las TiCs están tan enraizadas en la actualidad que no basta con ser un usuario más, sino que todo estudiante y egresado de universidad debe adicionalmente dominar las TICs de su especialidad.

Desde esta perspectiva, la propuesta que aquí se plantea es establecer como una competencia específica de cada carrera el uso experto de las TICs según la especialidad. 
Por ejemplo, para un docente, la competencia correspondiente podría ser esta: Capacidad para dominar con experticia las tecnologías de información y comunicación enfocadas en el aprendizaje y la enseñanza de las asignaturas de su especialidad.

\subsection{Tercer reto: la nueva concepción del perfil profesional sustentado en competencias y metas educativas}

El perfil profesional del egresado de la carrera no solo deberá sustentarse en el nuevo paradigma y las nuevas metodologías pedagógicas, sino que, además, deberá tener como interlocutores valiosísimos a los mercados laborales local y global. Este desafío atañe directamente a los gestores educativos y a los docentes; y las implicancias que tienen sobre el futuro egresado de las carreras universitarias son, dicho de la forma más sencilla, capitales.

La nueva concepción del perfil profesional entraña, por lo menos, dos consecuencias cardinales para la gestión y el ejercicio de la docencia. La primera está relacionada con la competencia de la empleabilidad. La segunda consecuencia exige una nueva forma de encarar la profesión de docente universitario (dicho sea entre paréntesis, una tercera consecuencia es que las carreras deban cumplir con las exigencias de políticas educativas nacionales e internacionales, pero, en este artículo, reservamos ese apartado para el quinto reto).

\subsubsection{La empleabilidad como competencia genérica}

Con respecto a la competencia de empleabilidad, esta aparece una y otra vez en los inicios del proceso de Bolonia. En la Declaración de Bolonia (1999) se cita la Declaración de La Sorbona (1998): "Se hizo hincapié en la construcción del espacio europeo de enseñanza superior como instrumento clave en la promoción de la movilidad de los ciudadanos, su ocupabilidad y el desarrollo global del continente" (las cursivas son nuestras). En el Comunicado de Londres (2007), se solicita al Grupo de Seguimiento de Bolonia "que estudie con todo detalle cómo incrementar la empleabilidad en cada uno de los tres ciclos [licenciatura, maestría, doctorado], así como en el contexto del aprendizaje a lo largo de la vida" (las cursivas son nuestras). Por esa razón, sorprende que entre las veintisiete competencias genéricas "acordadas" para América Latina (Beneitone et al., 2007, pp. 44-45) por Tuning América Latina no figure la empleabilidad. Tampoco aparece en la siguiente publicación global de Tuning América Latina (Beneitone et al., 2014).

La Real Academia Española (RAE) y la Asociación de Academias de la Lengua Española (ASALE) definen la empleabilidad como el "conjunto de aptitudes y actitudes que permiten a una persona conseguir y conservar un empleo" $(2014,2018)$. Nótese que se trata de una buena definición, que excluye o supone los conocimientos, pero que brinda explícita presencia a las habilidades (aptitudes) y a los valores asociados (actitudes). Sin embargo, no da cabida a la competitividad en el oficio, pues limita la competencia a "conseguir y conservar un empleo"; y no refiere el potencial de la persona a cambiarse a otra organización con mejor estatus. Hoy la empleabilidad sigue siendo un horizonte ansiado. Incluso, en referencia a un sistema de educación superior tan relevante como es el de Chile, se indica que aún existe "una preocupación seria sobre la relación de gran parte de los resultados de la educación terciaria, con las necesidades del mercado laboral, especialmente en el sector universitario" (OCDE y Banco Internacional para la Reconstrucción y el Desarrollo/Banco Mundial, 2009, p. 313).

¿Es la empleabilidad una competencia de la mayor importancia para figurar como una competencia genérica de la educación superior en América Latina? Aseguramos que sí y es esta la primera propuesta para el tercer reto. Precisamente, es el perfil del profesional, sustentado en competencias y metas educativas, el que debe asegurar la consecución eficaz de la competencia de empleabilidad.

Resulta importante señalar, en términos de lo dicho hasta ahora, que la competencia de empleabilidad va de la mano con la competencia de aprender a aprender. Si las universidades sólo persiguieran egresados empleables, la empleabilidad de estos decaería con el transcurrir del tiempo, pues una característica esencial de nuestra época es el cambio permanente. Por ello, un egresado empleable que, además, detenta la competencia de aprender a aprender, afrontará con mucho mayor éxito la incertidumbre y el cambio; y renovará de forma permanente sus competencias 
para seguir siendo empleable. Pero, para ello, también es necesario reformular la profesión del docente universitario.

\subsubsection{Una nueva forma de encarar la profesión de docente universitario}

Aun cuando el problema no es exclusivo del Perú, sino que se extiende por toda América Latina, es preciso dejar constancia de una grave falencia que subsiste en el sistema peruano de educación superior universitaria, falencia que no ha sido satisfecha y acaso ni siquiera advertida por la reciente normativa sobre el tema (Congreso de la República del Perú, 2014; Minedu, 2015a, 2015b; Sineace, 2016, 2017). Incluso admitiendo que "la cultura de la calidad educativa sí esta presente [en el Perú actual], y en las instituciones en las que ha llegado ya ha generado cambios que seguramente no tendrán regreso" (Sevillano, 2017), es fundamental subrayar esta falencia; la gran mayoría de futuros profesores universitarios, cuando acceden a la docencia universitaria, son profesionales especializados en una determinada carrera, pero no son profesores.

En el transcurso de su carrera, algunos de estos profesionales especializados, de forma voluntaria, optan por complementar su carrera profesional con la carrera pedagógica (circunstancia ideal); otros se limitan a cumplir con las capacitaciones de didáctica y pedagogía que la universidad fija de forma obligatoria (en el caso de que, efectivamente, la universidad fije esto); y algunos pocos, a partir de esas horas anuales y de la experiencia adquirida, llegan a ejercer la docencia con relativa eficiencia. Creemos que muchos tienen pocos conocimientos de índole pedagógica para afrontar los procesos de aprendizaje y enseñanza en el aula, lo cual configura un panorama sombrío, desde un punto de vista de eficacia como de eficiencia.

A partir de la nueva ley universitaria, dada a conocer en 2014 (Congreso de la República del Perú, 2014), se exige que quienes enseñen en pregrado deben tener, como mínimo el grado de magíster o maestro y los que enseñen en posgrado con el grado de doctor. En ninguna de las leyes, decretos o reglamentos dados en los últimos años se solicita que el docente universitario deba contar con un grado de educación cuando, por sentido común, si hay una labor que un docente universitario ejerce es, precisamente, la docencia; y las autoridades y expertos en educación peruanos e internacionales-los textos del Sineace $(2016,2017)$ señalan la participación de consultores internacionales-en lo que menos han pensado es en solicitar que el docente universitario sea docente de formación.

La segunda propuesta consecuente para el tercer reto es que el docente universitario, además de haber obtenido el grado y el posgrado correspondientes en su carrera y especialidad, debe contar con un título de profesor o docente de forma obligatoria. Esta propuesta, además, debería de extenderse no sólo a los docentes, sino a todos aquellos que, sin ejercer la docencia, influyen de forma gravitante en los procesos de aprendizaje. De esta forma, toda persona involucrada directamente en procesos de aprendizaje, enseñanza y gestión de la educación superior contaría con título de profesor o docente, desde el tutor, ayudante de cátedra o jefe de práctica hasta los decanos, el rector y los que estuvieran en una posición de gestión superior a los decanos y al rector, pasando, claro está, por los docentes, coordinadores de curso, jefes de área, directores, etcétera. Desde esta perspectiva, todos los involucrados en educación sabrían en qué consiste ésta y no sólo estarían capacitados para contribuir en la elaboración eficiente de un adecuado perfil profesional, sino que también estarían habilitados para la eficaz realización de ese perfil.

\subsection{Cuarto reto: la asunción del paradigma que coloca en el centro del proceso enseñanza-aprendizaje al estudiante y relega al profesor en el rol de facilitador}

La asunción de este paradigma trae como consecuencia lo siguiente: que en las instituciones de educación superior se asuma el enfoque por competencias como el más adecuado y coherente con el paradigma mencionado. ¿Por qué el enfoque por competencias? Por las tres razones que se analizan a continuación.

\subsubsection{Primera razón: porque es altamente efectivo} En principio, el enfoque por competencias es el primer tipo de metodología que ha demostrado ser altamente efectivo en procesos de enseñanzaaprendizaje con grupos amplios e, incluso, masivos de estudiantes. Algunos otros enfoques, como, por ejemplo, el del superaprendizaje (Ostrander, Ostrander \& Schroeder, 1981, 1989), sólo han sido implementados 
en contextos experimentales o con grupos reducidos. Más aún, es necesario señalar que "la formación profesional tradicional, basada en contenidos y el credencialismo, pareciera no ser capaz de responder efectivamente a esta demanda [profesionales capaces de resolver, con un desempeño ético, diferentes problemas de la especialidad]" (Cinda, 2010, p. 16). En cambio, por aprendizaje altamente efectivo debe entenderse lo que también se conoce como aprendizaje auténtico, es decir, aquel aprendizaje que se realiza o demuestra en la acción. Así, si en la unidad académica se ha fijado como logro que el estudiante, al finalizarla, debe elaborar un informe de auditoría ambiental, el aprendizaje auténtico del estudiante se traducirá en la elaboración efectivamente autónoma y eficiente de un informe de auditoría ambiental.

\subsubsection{Segunda razón: porque contribuye con la empleabilidad}

La segunda razón es que el enfoque por competencias logra en los educandos las capacidades necesarias para desempeñarse eficientemente en el entorno laboral o profesional inmediato. En otras palabras, el enfoque por competencias es el que asegura mayor índice de empleabilidad de los futuros egresados.

\subsubsection{Tercera razón: porque es coherente con la formación de expertos}

La tercera razón es que el enfoque por competencias guarda coherencia científica; se halla correctamente sustentado con las investigaciones sobre la cognición de la experticia. Desde principios de la década de 1990, K. Anders Ericsson y los que bajo su liderazgo investigaban sobre la experticia en diferentes dominios habían empezado a llegar a conclusiones que, años después, demostrarían y generalizarían en todo ámbito del quehacer humano: se requieren no menos de 10,000 horas en un periodo de aproximadamente diez años, utilizando técnicas metodológicas como la práctica deliberada y el acompañamiento de mentores, para que una persona adquiera la experticia en determinado dominio (Ericsson, Krampe \& TeschRömer, 1993; Ericsson, Prietula \& Cokely, 2007; Ericsson, 2009; Ericsson \& Smith, 1991; Ericsson, Charnes, Feltovich \& Hoffman, 2006). En otras palabras, en las investigaciones cognitivas de Ericsson y colaboradores se ha llegado a la conclusión de que, para formar expertos, se requiere de procesos formativos concentrados en la práctica intensa y deliberada y de cara a las metas que el dominio requiera de la experticia; y lo más cercano a esa metodología en educación es el enfoque por competencias.

\subsection{Quinto reto: el alcance global de la actividad humana que implica la internacionalización de la educación superior}

El fenómeno de esta última globalización es la macrotendencia universal que define nuestra contemporaneidad. Actualmente la actividad humana, por muy alejada o periférica que se halle respecto de los centros de poder, tiene alcance global. Lo mismo pasa con la educación. Y, por ello, el quinto reto, que implica de por sí solo su propuesta consecuente, es la internacionalización de la educación, con lo cual se regresa a las fuentes del proceso de Bolonia: movilidad geográfica, cultural y académica de estudiantes y docentes, más calidad y competitividad, más diversidad e inclusión. En una palabra, internacionalización.

Sin embargo, la internacionalización como reto y respuesta a ese mismo reto puede parecer una respuesta imprecisa. Por esa razón, la respuesta más específica al reto que implica la internacionalización es el cumplimiento cabal de las políticas educativas globales y locales (idealmente, en ese orden). Y esa es, en suma, nuestra propuesta consecuente al quinto reto.

\subsection{1. ¿Qué implican esas políticas educativas?}

Se trata de políticas de alcance internacional y nacional, normativas y vinculantes para todas las instituciones implicadas. En el caso del Perú, expresan políticas educativas, como la ley universitaria de 2014; la política de licenciamiento de las universidades por la Superintendencia Nacional de Educación Superior Universitaria (Sunedu); los decretos del Ministerio de Educación (Minedu), los sistemas de aseguramiento de la calidad o de acreditación del Sistema Nacional de Evaluación, Acreditación y Certificación de la Calidad Educativa (Sinease). Los procesos de armonización de los sistemas de educación superior poseen carácter internacional. Los sistemas de créditos transferibles y los marcos de cualificación también tienen alcance supranacional.

Se debe señalar que, a partir de la promulgación de la 


\section{REVIEW}

OF GLOBAL

\section{MANAGEMENT}

FACULTAD DE

NEGOCIOS

UPC

ley universitaria de 2014, las universidades peruanas han uniformizado el número de créditos académicos de sus carreras. Se ha establecido un mínimo de 200 créditos académicos, de los cuales 35 corresponden a estudios generales, y 165 a estudios específicos y de la especialidad, en concordancia con los artículos 41 y 42 de la nueva ley universitaria, i.e., Ley 30220 (Congreso de la República del Perú, 2014).

Huelga decir que el sistema educativo de cualquier universidad peruana es tributario obligado del sistema nacional peruano, el cual tendría que establecer criterios comunes con las instituciones de América Latina que se propondrían seguir las equivalencias puntuales del proyecto Tuning, a fin de que las carreras puedan ser homologables y convalidables. Sin embargo, el sistema educativo peruano no ha tenido en cuenta la experiencia de Tuning América Latina.

\subsubsection{El sistema de créditos transferibles}

Como ya se ha dicho antes, una de las ideas centrales que inspiró el proceso de Bolonia fue la internacionalización de la educación. Dicha aspiración implicaba que los planes educativos de las universidades fueran homologables y, por tanto, convalidables. Para ello, los programas educativos debían estar armonizados en sus contenidos, logros y competencias por alcanzar, lo que implicaba que los programas y las carreras se insertaban en el ECTS (Sistema Europeo de Transferencia y Acumulación de Créditos), además de la adopción de la estructura de tres ciclos: licenciatura, maestría y doctorado (Gimeno, 2004).

En América Latina, y con mayor precisión en el sistema de educación superior de Chile (Universidad Andrés Bello de Chile, s. f.; Kri Amar et al., 2013), ya es una realidad el SCT-Chile (Sistema de Créditos Académicos Transferibles-Chile). En el Perú aún no se cuenta con un "SCT-Perú"; por ahora, solo se tiene un modelo de acreditación para programas de estudios de educación superior universitaria (Sineace, 2016).

\subsection{3. ¿Qué implicaría contar con un sistema de créditos transferibles?}

En primer lugar, los primeros implicados son las autoridades a cargo del sistema de la educación; a saber, en el caso del Perú, el Minedu y la Sunedu. Se debe señalar que la promulgación de la nueva ley universitaria, de 2014, Ley 30220, fue una oportunidad perdida para que el sistema de educación superior peruano se insertara en la dinámica internacional. Lejos de ello, la nueva ley universitaria cabalga en ámbitos locales y, como ya se dijo antes, establece un mínimo de 200 créditos académicos por carrera, de los cuales 35 corresponden a estudios generales y 165 a estudios específicos y de la especialidad (artículos 41 y 42 de la nueva ley universitaria) (Congreso de la República del Perú, 2014). Hay que agregar que en la nueva ley universitaria aún se habla de estudios generales, específicos y de la especialidad, y no se asume el idioma hoy universal de las competencias genéricas y específicas.

El sistema de Chile, más acorde con el sistema internacional, establece que en una carrera de pregrado son necesarios 60 SCT-Chile por año, lo que implica entre 24 y 31 horas por cada SCT-Chile, lo cual suma entre 1440 y 1900 horas cronológicas anuales, aproximadamente. En cinco años, un alumno chileno logrará 300 SCT-Chile y un aproximado de 9500 horas cronológicas. Tomando las ideas de Ericsson y otros autores, tendremos que el egresado chileno está mucho más cerca de lograr la experticia (10,000 horas en un periodo de 10 años), que un egresado peruano.

Es innecesario mencionar que asumir un sistema de créditos transferibles también implica, es decir, involucra, a docentes y estudiantes. Los docentes deben tener claras las exigencias que las asignaturas homólogas del sistema (europeo, americano [de América, no de Estados Unidos] o latinoamericano) plantean, a fin de que las asignaturas locales sean homologables y convalidables a partir de estándares previamente establecidos.

Una vez claras esas exigencias macro, los docentes deben diseñar sus asignaturas teniendo en cuenta las horas efectivas que el estudiante empleará en el aprendizaje del curso. En esas horas efectivas se incluyen no sólo las horas de clase lectivas, las horas de aprendizaje autónomo y las de actividades virtuales u online, sino también todas aquellas horas que el estudiante invertirá en prepararse para desempeñarse 
adecuadamente en las evaluaciones.

Asimismo, los estudiantes deberían tener claro que cada curso exige de ellos un aproximado número de horas por unidad académica, por semana, por lección. Esto exige que no sólo los docentes, sino también los estudiantes, gestionen su propio aprendizaje y se conviertan en gestores efectivos de tiempo.

\subsubsection{Beneficios de contar con un marco de cualificación}

Para el sistema de educación superior peruano, el mayor beneficio que implicaría contar con un marco de cualificación como el europeo o el chileno sería la movilidad educativa de alumnos y de docentes. Insertos en un sistema internacional (europeo, americano o latinoamericano), las carreras, programas y cursos de las universidades peruanas serían homologables y convalidables. Un estudiante peruano podría llevar cursos en el Perú o en cualquiera de los países del mismo sistema. El estudiante peruano adquiriría el estatus de estudiante internacional y podría movilizarse de manera fluida de una universidad a otra, de ser admitido como alumno. Lo mismo ocurriría con los docentes peruanos: ellos contarían con las capacidades para laborar en una universidad y otra pertenecientes al mismo sistema.

Un beneficio subordinado a la movilidad académica horizontal sería el de la estandarización de los cursos. Esto implica, tanto la calidad (de diseño, de metodologías, de materiales), como la cantidad (de créditos académicos transferibles, de horas efectivas de trabajo académico), que cada asignatura implicaría.

\section{CONCLUSIÓN}

Al asumir las cinco tendencias universales establecidas por Tuning América Latina en 2007 (Beneitone et al., 2007), como retos que la gestión y el ejercicio de la docencia deben afrontar, entendemos que esas tendencias-retos sintetizan bien la problemática actual de los procesos de aprendizaje en la educación superior en el mundo, pero con énfasis especial en aquellos que se realizan en América Latina. Desde esta perspectiva, es necesario señalar que somos conscientes de que el horizonte en nuestro continente acaso no posea los perfiles homogéneos de Europa, ni posiblemente la decisión de consenso de las autoridades políticas y educativas de países signados por una heterogeneidad histórica, social y económica. El trabajo se avizora arduo y tal vez heteróclito, pero, por lo mismo, se vuelve más desafiante y estimulante.

\section{REFERENCIAS}

Beneitone, P., Esquetini, C., González, J., Maletá, M. M., Siufi, G. \& Wagenaar, R. (Eds.) (2007). Reflexiones y perspectivas de la educación superior en América Latina. Informe Final-Proyecto Tuning-América Latina 2004-2007. Bilbao: Universidad de Deusto y Universidad de Groningen.

Beneitone, P., González, J. \& Wagenaar, R. (Eds.) (2014). Meta-perfiles y perfiles. Una nueva aproximación para las titulaciones en América Latina. Bilbao: Universidad de Deusto.

Bugarin Olivera, R. (2008). Educación superior en América Latina y el proceso de Bolonia: alcances y desafíos. CienciaUAT, 3(2), 54-58.

Centro Interuniversitario de Desarrollo (Cinda). (2010). Diseño curricular basado en competencias y aseguramiento de la calidad en la educación superior. Santiago de Chile: Autor.

Comunicado de Londres. (2007). Hacia el Espacio Europeo de Educación Superior: respondiendo a los retos de un mundo globalizado (Material didáctico publicado por la Universidad Andrés Bello de Chile).

Congreso de la República del Perú. (2014). Ley 30220. Ley Universitaria. Recuperado de https://www. sunedu.gob.pe/wp-content/uploads/2017/04/ Ley-universitaria-30220.pdf 


\section{REVIEW}

OF GLOBAL

\section{MANAGEMENT}

FACULTAD DE

NEGOCIOS

UPC

Declaración de Bolonia. (1988). Bolonia y la Magna Charta Universitatum (Material didáctico publicado por la Universidad Andrés Bello de Chile).

Declaración de Bolonia. (1999). Declaración conjunta de los ministros europeos de Educación (Material didáctico publicado por la Universidad Andrés Bello de Chile).

Declaración de La Sorbona. (1998). Declaración conjunta para la armonización del diseño del Sistema de Educación Superior Europeo (Material didáctico publicado por la Universidad Andrés Bello de Chile).

Ericsson, K. A., Krampe, R. T. \& Tesch-Römer, C. (1993). The role of deliberate practice in the acquisition of expert performance. Psychological Review. 100(3), 363-406.

Ericsson, K. A., Prietula, M. J. \& Cokely, E. T. (2007). The making of an expert. Harvard Business Review. Recuperado de https://hbr.org/2007/07/themaking-of-an-expert

Ericsson, K. A. (Ed.) (2009). Development of professional expertise. Toward measurement of expert performance and design of optimal learning environments. Cambridge, New York: Cambridge University Press.

Ericsson, K. A. \& Smith, J. (Eds.) (1991). Toward a general theory of expertise: Prospects and limits. Cambridge, England: Cambridge University Press.

Ericsson, K. A., Charnes, N., Feltovich. P. J. \& Hoffman, R. R. (Eds.) (2006).The Cambridge handbook of expertise and expert performance. Cambridge, New York: Cambridge University Press.

Gimeno, J. (2004). El crédito europeo: un reto para la calidad de la enseñanza en la universidad (Documento de Trabajo del Proyecto Andaluz de Formación del Profesorado Universitario de la Unidad para la Calidad de las Universidades Andaluzas, 520-552) (Material educativo publicado por la Universidad Andrés Bello de Chile).
Kri Amar, F., Marchant Mayol, E., Del Valle Martin, R., Sánchez Doberti, T., Altieri Missana, E., Ibarra Palma, P .,..., Segovia Ormazábal, N. (2013). Manual para la implementación del sistema de créditos académicos transferibles SCT-Chile. Santiago de Chile: Consejo de Rectores de las Universidades Chilenas, CRUCH.

Ministerio de Educación del Perú. (2015a). Lineamientos de política para el aseguramiento de la calidad en la educación superior universitaria: aportes y recomendaciones. Recuperado de http:// franciscosagasti.com/site/wp-content/uploads/ 2015/07/BBBB-Lineamientos_politica-final.pdf

Ministerio de Educación del Perú. (2015b). D. S. 0162015 MINEDU. Aprobación de la Política de Aseguramiento de la Calidad de la Educación Superior Universitaria. Recuperado de https:// cdn.www.gob.pe/uploads/document/file/ 105041/_016-2015-MINEDU_-_21-10-2015_04_19_ 56_-DS_N_016-2015-MINEDU.pdf

Organización para la Cooperación y el Desarrollo Económicos \& el Banco Internacional para la Reconstrucción y el Desarrollo/Banco Mundial. (2009). La educación superior en Chile. Santiago de Chile: Autores.

Ostrander, S., Ostrander, N. \& Schroeder, L. (1981/1989). Superaprendizaje. Nuevos métodos para potenciar su memoria y mejorar su eficacia profesional y deportiva (8ª ed.) (Traducción de J. M. Álvarez Flórez \& A. Pérez). Barcelona: Grijalbo (Publicación original en inglés: 1981).

Real Academia Española \& Asociación de Academias de la Lengua Española. (2014/2018). Diccionario de la lengua española (23. ${ }^{\mathrm{a}}$ ed.). Recuperado de https://dle.rae.es/?id=ErTAI6H

Sanjurjo Rivo, V. A. (2012). El nuevo espacio europeo de educación superior: desafíos e incertidumbres. Revista de Derecho UNED, 10, 585-609. 
Sevillano, S. (2017). El sistema de acreditación universitaria en el Perú: marco legal y experiencias recientes. Revista de Educación y Derecho, 15. Recuperdo de http://revistes.ub.edu/index.php/RED/article/ view/18400/20952

Sistema Nacional de Evaluación, Acreditación y Certificación de la Calidad Educativa del Perú. (2016). Modelo de Acreditación para Programas de Estudios de Educación Superior Universitaria. Lima: Autor.

Sistema Nacional de Evaluación, Acreditación y Certificación de la Calidad Educativa del Perú. (2017). Mode lo de Acreditación Institucional para Universidades. Lima: Autor.

Toffler, A. (1979/1995). La tercera ola (11. a ed.) (Traducción de A. Martín). Barcelona: Plaza \& Janés. (Publicación original en inglés: 1979).

Universidad Andrés Bello. (s. f.). El sistema de créditos transferible (Material didáctico interactivo publicado por la Universidad Andrés Bello de Chile). 